\title{
Traitement non-opératoire des ulcères perforés
}

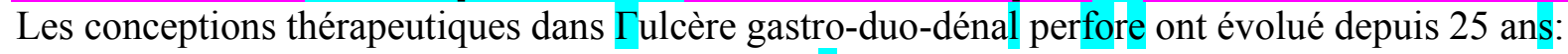
après avoir préconisé la suture, le nettoyage soigne de la cavité péritonéale et des drainages multiples, on a constate d'abord que les capacités phagocytaires du péritoine et la stérilité fréquente du liquide épanché permettaient de supprimer ces deux derniers temps, puis que la perforation se « oouvrait» d'elle-même la plupart du temps.

Ceci a amené E. W. Bedford-Turner (Brit. Med. J., 1, 457, 1945) à proposer uin traitement conservateur consistent en morphine pour calmer la douleur, lavage de $\Gamma$ estomac, aspiration permanente. Ces manoeuvres ont été codifíées par H. Taylor (Lancet, 2, 441, 1946), après Texpérienee de 28 cas, per-mettant aussi d'en préciser les indications.

La technique oonsiste en:

$1^{\circ}$ Injection intraveineuse de 0,015 de morphine, et faire sucer une pastille de cocaine pour faciliter le passage du large tube de Faucher, nécessaire à l'évacuation du contenu gastrique. $2^{\circ}$ Aspiration toutes les demi-heures le premier jour, toutes les heures le second jour, par la sonde d'Einhorn à demeure, in, troduite par le nez.

$3^{\circ}$ Réhpdratation du malade par voie parentérale ou $\Gamma$ ectale, en introduisant la quantité de liquide retiree de l'esto-mac plus $11 / 2$ à 2 litres. Dès le troisième jour, on peut faire bo $1 / 8 \mathrm{e}$ un peu de lait coupe.

Les résultats de Taylor sont sensationnels: 24 guérisons sans incidents, 3 décès pour des raisons non digestives, une seule péritonite par réouverture de $\Gamma$ ulcère colmaté chez un sujet indiscipline, ayant bu après la perforation. Ajoutons que deux cas ont dû être opérés secondairement, la contracture abdominale persistant. Le traitement fut entrepris chez 25 de ces malades entre la premiere et la sixième heure après $\Gamma$ acci-dent aigu, et il s'agissait toujours d'ulcères du duodenum. La statistique plus restreinte de A. H. Visick (Brit. Med. J., 2, 941,1946) comporte 14 cas, dont 1 ulcère gastrique (qui mou-rut d'ailleurs) et un ulcère peptique jéjunal; il y eut 3 décès, 